\title{
El papel de los profesionales en centros de atención en drogas en ambulatorios de la ciudad de Bogotá, Colombia
}

\author{
Luz Patricia Díaz Heredia ${ }^{1}$ \\ Maria Helena Palucci Marziale ${ }^{2}$
}

Se trata de un estudio descriptivo y trasversal, desarrollado con 93 profesionales, que tuvo por objetivo caracterizar el papel de los profesionales que se desempeñaban en los centros de atención en drogadicción en ambulatorios de la ciudad de Bogotá, Colombia. Los resultados mostraron que los profesionales que se encontraron con mayor frecuencia fueron los psicólogos (38\%) y los que desempeñaban actividades terapéuticas eran el 34\%. Se evidencia que los enfermeros, que correspondieron al 8\% del profesionales, enfatizaron su papel en el ámbito administrativo (43\%) y predominantemente en los aspectos de promoción del auto cuidado (100\%), prevención (57\%) y muy poco en la rehabilitación y reinserción social (14\%). Los papeles desempeñados por los profesionales coinciden con lo esperado a respecto de las actividades de las diferentes profesiones, con excepción de los terapeutas ocupacionales. Se considera que las enfermeras necesitan ganar más espacio dentro del equipo de atención a los usuarios de drogas.

Descriptores: Rol Profesional; Enfermería; Trastornos Relacionados con Sustancias; Alcoholism.

\footnotetext{
Corresponding Author:

Maria Helena Palucci Marziale

Universidade de São Paulo. Escola de Enfermagem de Ribeirão Preto

Av. Bandeirantes, 3900

Bairro Monte Alegre

CEP: 14040-902 Ribeirão Preto, SP, Brasil

E-mail: marziale@eerp.usp.br
}

${ }^{1}$ Enfermera, Profesor Asociado, Universidad Nacional de Colombia, Colombia. E-mail: Ipdiazh@unal.edu.co.

2 Enfermera, Doctor en Enfermería, Profesor Titular, Escola de Enfermagem de Ribeirão Preto, Universidade de São Paulo, Centro Colaborador de la OMS para el Desarrollo de la Investigación en Enfermería, SP, Brasil. E-mail: marziale@eerp.usp.br. 


\section{Papéis de profissionais, em centros de atenção ambulatorial em drogas, na cidade de Bogotá, Colômbia}

Estudo descritivo, transversal, desenvolvido com 93 profissionais. O objetivo foi caracterizar o papel e as atividades desempenhadas pelos profissionais atuantes nos centros de atenção ambulatorial a usuários de drogas, da cidade de Bogotá, Colômbia. Os resultados mostraram que os profissionais atuantes em maior número foram os psicólogos (38\%), desempenhando atividades em comunidades terapêuticas (34\%). Evidenciou-se que os enfermeiros, que correspondem a $8 \%$ dos profissionais, enfatizaram seu papel em atividades administrativas (43\%) e, predominantemente, nos aspectos de promoção do autocuidado $(100 \%)$, prevenção $(57 \%)$ e pouco na reabilitação e reinserção social (14\%). O papel desempenhado pelos profissionais coincide com o esperado em relação às condutas e atividades desempenhadas pelas diferentes profissões, com exceção dos terapeutas ocupacionais. Considera-se que os enfermeiros precisam conquistar maior espaço dentro da equipe de atenção aos usuários de drogas.

Descritores: Papel Profissional; Enfermagem; Transtornos Relacionados ao Uso de Substâncias; Alcoolismo.

\section{Roles of Professionals in Drug Outpatient Care Centers, in the City of Bogota, Colombia}

The aim of this descriptive, cross-sectional study, developed with 93 professionals, was to characterize the roles of the professionals who were working in the drug addiction outpatient centers of the city of Bogota, Colombia. The results showed that psychologists were the predominant professionals (38\%), followed by occupational therapists $(34 \%)$. Nurses corresponded to $8 \%$ of the professionals, with their roles predominantly in the aspects of health promotion and self care (100\%), in the administrative area (43\%) and in prevention (57\%) and very little in rehabilitation and social rehabilitation (14\%). The roles of the professionals agreed with the expected activities of the different professions, with the exception of the occupational therapists. It is considered that nurses need to gain more space within the team to focus on drug users.

Descriptors: Professional Role; Nursing; Substance-related Disorders; Alcoholism.

\section{Introducción}

Esta investigación parte del compromiso que tienen los profesionales de la salud frente al fenómeno de las drogas, que en Colombia, como en América Latina, ha evolucionado transformándose en una problemática social, de salud pública, política y económica. Algunas particularidades de esta compleja situación se relacionan con el tratamiento y las intervenciones realizadas por los profesionales que atienden a las personas que vivencian situaciones de adicción.

En Colombia, estos asuntos son de gran importancia, debido a gran disponibilidad de las sustancias y a las inequidades de índole social y económica que han precipitado el incremento de la incidencia y prevalencia del consumo, primordialmente en los grupos poblacionales jóvenes ${ }^{(1-5)}$. Respecto a las adicciones, el efecto del consumo abusivo lleva a repercusiones graves de la salud, física, emocional y social, lo cual requiere del establecimiento ofrecer una rehabilitación y tratamiento integral, comprensivo, oportuno y eficaz, por parte de los diferentes profesionales que están involucrados en la atención a la persona que enfrenta una situación de adicción.

Al verificar los índices de consumo, que son cada día mayores, se deduce que se necesita un mayor número 
de profesionales y de instituciones idóneas para tratar y rehabilitar la adicción a sustancias psicoactivas, tanto lícitas como ilícitas. Se requiere de la asistencia y apoyo de los profesionales de las ciencias biológicas y sociales para enfrentar y sobrepasar la adicción a las sustancias psicoactivas. En Colombia, el consumo se sustancias licitas e ilícitas está llegando a niveles alarmantes, en especial el alcohol que sobrepasa el $80 \%$ de prevalencia de vida en la población en general(2).

En el último estudio epidemiológico nacional, se reportó que el alcohol es la sustancia más consumida en el país, entre el 70 y el $90 \%$ de la población ha consumido esta sustancia alguna vez en la vida. En los niños y adolescentes puede verse como la escolaridad es un factor protector para el consumo, ya que los niños que abandonan la escuela tienen un riesgo tres veces mayor de iniciarse en el consumo; también los adolescentes que se atrasan escolarmente tienen 4,4 veces más riesgo de usar drogas frente a aquellos que no repiten $\operatorname{años}^{(3)}$.

El Ministerio de la Protección Social en el año 2007, refiere que entre un 9 a un $10 \%$ de los estudiantes están consumiendo drogas lícitas (alcohol o tabaco) e ilícitas; el $80 \%$ por ciento de los estudiantes en Colombia está consumiendo alcohol y cerca del 46\% consume tabaco, según el estudio realizado en el año anterior con estudiantes de grado básico secundario y universitarios. En el caso de las ilegales, la marihuana sigue en el primer lugar de consumo, con el $8 \%$, seguida de la cocaína, con el $7 \%$. Pero es preocupante que el $9 \%$ de estudiantes acepten que ha consumido "drogas de control" o bajo prescripción médica, las drogas de laboratorio, como el éxtasis, son consumidas por cerca del $2 \%$ de los entrevistados. El consumo de drogas en Colombia viene, en un preocupante, ascenso; la edad media de inicio de la población está cerca de los once años ${ }^{(6)}$.

El Ministerio de la Protección Social y la Oficina de las Naciones Unidas sobre Drogas, UNODC, realizaron una consulta nacional sobre la situación del país en materia de uso indebido de sustancias psicoactivas(5), la cual indicó, que tal como se había reportado en los estudios anteriores $y$, al igual que en muchos otros escenarios en el mundo, las sustancias de más alto consumo son el alcohol y el cigarrillo, seguidas por la marihuana, que aparece como la sustancia ilícita más consumida en ambos sexos y en todos los estratos socioeconómicos. Aunque los inhalables y los tranquilizantes presentan, después de la marihuana, los más altos índices de consumo entre todas las sustancias ilícitas en la población general, la cocaína continúa siendo la segunda sustancia ilícita más consumida entre los adultos mayores de 30 años, mientras que el éxtasis se posiciona paulatinamente como la segunda sustancia ilícita entre adultos jóvenes y adolescentes de ambos sexos, de estratos socioeconómicos medio y alto, en tanto que en ámbito de Sudamérica se ubica como el más elevado con 4,9\% de prevalecía en la vida(1,5).

Por otra parte los datos presentados por el sistema de Vigilancia Epidemiológica para Sustancias Psicoactivas(2), reflejan aspectos epidemiológicos relacionados con la atención hospitalaria a los consumidores. Durante 1998, en 49 instituciones hospitalarias se atendieron 1.851 casos relacionados con consumo de sustancias psicoactivas. Para el primer trimestre de 2005 se informa de 1.223 casos de abuso en centros especializados en el tratamiento de la farmacodependencia. Aparentemente se presenta un importante incremento en la demanda de servicios hospitalarios por esta causa, $81,6 \%$ de los usuarios eran hombres, mientras 18,4 eran mujeres. Para 2005, el $75,6 \%$ correspondió a los primeros y el $24,4 \%$ a las segundas. Los desempleados, con 39\%, fueron los mayores usuarios, seguidos de trabajadores fijos $y$ ocasionales con $32 \%$ y estudiantes con $29 \%$.

Para dar atención de calidad a las personas que presentan situaciones de adicción en el contexto colombiano, se han establecido normas como la resolución 196 del 26 de febrero de 2002(3), por medio de la cual se establecían las normas técnicas, científicas y administrativas para el funcionamiento de los centros de atención, tratamiento y rehabilitación integral. En esta norma se encontraban claramente definidos los requisitos de recurso humano, de recurso material y físico requeridos para la atención, así como las orientaciones respecto a las actividades y funciones que se deben desarrollar en los diferentes niveles de atención, aspectos que, en las actuales normas no se encuentran contenidas, por lo que las actividades y papeles se encuentran desdibujados en lo cotidiano y no es claro quienes las desarrollan.

Surge la inquietud de conocer cuáles son las actividades que denotan el papel desempeñado por el profesional en las instituciones que atienden personas con adicción a sustancias psicotrópicas en los centros de atención en drogas en ambulatorios en la ciudad de Bogotá. Se parte del concepto de este papel desde dos perspectivas, la primera referida a la visión antropológica sociológica. Esta entiende los papeles como una agrupación de valores, actitudes y conductas que la sociedad asigna a las personas que ocupan una 
posición ligada a un determinado status social. La segunda perspectiva englobaría la visión sicosocial, en donde se resaltan los contenidos y conductas esperadas en relación a una posición laboral concreta(7). Visto de esta forma el papel ubica a la persona en un contexto social determinado y en una estructura social y grupal concreta. Por otra parte es necesario conocer si los profesionales están formados en el área en la cual se encuentran desempeñándose, ya que se requiere promover la formación en este sentido y en esta área del conocimiento en particular. Los profesionales de la salud y en especial las enfermeras no cuentan con conocimientos suficientes para enfrentar las situaciones de intervención en las situaciones de alteración mental y de adicción ${ }^{(8)}$.

Para el profesional de enfermería que se encuentra trabajando - como miembro del grupo interdisciplinario en las instituciones públicas, privadas o mixtas que presta servicios de salud en sus fases de tratamiento y rehabilitación, bajo la modalidad ambulatoria o residencial, a personas con adicción a sustancias psicoactivas, mediante la aplicación de un determinado modelo o enfoque de atención, basado en evidencia es necesario establecer si es ella quien se encuentra desarrollando las actividades de promoción y prevención y cuál es su participación en este nivel, ya que es ella quien hasta el momento ha desarrollado primordialmente estas actividades en otros países latinoamericanos, como se encuentra referido en la literatura de otras latitudes.

Teniendo en cuenta lo anterior, se evidencia la necesidad de contar con instituciones y profesionales de salud que desarrollen actividades de tratamiento y rehabilitación coherentes con las políticas y las normas establecidas por el Ministerio de Protección Social, ente encargado de su elaboración y promulgación. En vista de la importancia que reviste el asunto, es necesario conocer el papel que los profesionales desempeñan en los centros de atención en drogadicción en ambulatorios con el objetivo de desarrollar programas de capacitación, acordes con los requerimientos del actuar profesional; desarrollar modificaciones en los currículos de los programas de formación; y, adecuar las normas existentes en el país respecto a la acreditación y la calidad de atención en salud.

\section{Objetivo}

Caracterizar el papel de los profesionales que se desempeñaban en los centros de atención en drogadicción en ambulatorios de la ciudad de Bogotá, Colombia.

\section{Método}

Se trata de un estudio cuantitativo, descriptivo y trasversal; fue realizado en la ciudad de Bogotá, Colombia, en los centros de atención en drogas que ofrecen servicios ambulatorios (CAD). La muestra estuvo constituida por 93 profesionales que se encontraban desarrollando actividades en treinta Centros de Atención de Drogas, que ofrecían tratamiento en ambulatorios en las diferentes áreas geográficas de la ciudad de Bogotá.

Los datos de la investigación fueron recolectados a través de un cuestionario compuesto de preguntas objetivas y analíticas, que fue sometido a evaluación de su contenido, claridad y objetividad, por tres expertos en la temática de uso y abuso de alcohol y drogas. Se realizó una prueba piloto con cinco profesionales que trabajaban en servicios de salud mental de la ciudad de Bogotá.

El instrumento y el consentimiento informado fueron entregados personalmente a los profesionales de la salud de las instituciones de atención en ambulatorios, a través de visitas desarrolladas por dos auxiliares de investigación, las cuales fueron entrenadas en el manejo del formulario y en la recolección de la información. La recolección se realizó entre los meses de febrero y mayo de 2008 .

Para realizar el estudio, se obtuvo el previo consentimiento por parte de la institución, confirmado mediante la firma de aceptación por parte de los gerentes o representantes legales de las mismas. El proyecto fue aprobado por el Comité de Ética de Investigación de la Universidad Nacional de Colombia - Facultad de Enfermería.

Los datos fueron tratados y analizados mediante el paquete estadístico SPSS 15 y se obtuvieron medidas de tendencia central y dispersión para las variables discretas y continuas; para las variables categóricas se obtuvieron frecuencia y porcentaje. Los resultados se presentan mediante tablas y gráficos. Con el fin de fragmentar, comparar y clasificar los datos cualitativos de las preguntas abiertas se utilizaron las técnicas de análisis de contenido manifiesto(9).

\section{Resultados}

La muestra estuvo constituida por 93 profesionales con edad media de 31 años y cuya desviación estándar fue 6,94 años. La edad máxima fue de 55 años y la mínima de 21 años. El género, el estado civil, el tipo de vinculación y el tiempo de experiencia se presentan en la Tabla 1. 
Tabla 1 - Distribución por frecuencia y porcentajes de los profesionales que laboran en los Centros de Atención de Drogas de Bogotá, según estado civil, género, tipo de vinculación y tiempo de experiencia.

\begin{tabular}{lcc}
\multicolumn{1}{c}{ Variables } & $\mathbf{n}$ & $\%$ \\
\hline Estado civil & 35 & 38 \\
Casados & 55 & 59 \\
Solteros & 01 & 1 \\
Unión Libre & 02 & 2 \\
Divorciado & & \\
Género & 49 & 53 \\
Masculino & 44 & 47 \\
Femenino & & \\
Tipo de vinculación & 15 & 16 \\
Estudiante Postgrado & 78 & 84 \\
Empleado & & \\
Tiempo de experiencia & 60 & 65 \\
1 -3 años & 28 & 30 \\
3años 1 día a 6 años & 05 & 5 \\
6 años 1 día a 8 años & 93 & 100 \\
Total & &
\end{tabular}

Los resultados de las sustancias consumidas por los pacientes atendidos y los diagnósticos realizados se presentan en la Tabla 2.

Tabla 2 - Distribución por frecuencia y porcentajes según los diagnósticos médicos de los pacientes atendidos en los CAD de la ciudad de Bogotá y las sustancias psicoactivas referidas como causa de adicción

\begin{tabular}{lcc}
\multicolumn{1}{c}{ Variables } & $\mathbf{n}$ & $\%$ \\
\hline Diagnósticos médicos & & \\
Alteraciones psicoafectivas & 26 & 28 \\
Enfermedad psiquiátrica asociadas al consumo & 24 & 26 \\
Dependencia a sustancias psicoactivas & 31 & 33 \\
otros & 04 & 4 \\
Sin información & 9 & 10 \\
Sustancias psicoactivas consumidas & & \\
alcohol, cigarrillo, cocaína & 26 & 28 \\
alcohol, cigarrillo, marihuana & 23 & 25 \\
alcohol & 16 & 17 \\
cigarrillo & 15 & 16 \\
cocaína, bazuco (marihuana con cocaína) & 10 & 11 \\
otros & 03 & 3 \\
Total & 93 & 100 \\
\hline
\end{tabular}

En cuanto a las profesiones desempeñadas 38\% eran Psicólogos, 35\% Médicos, 8\% Enfermeras, 5\% Terapeutas Ocupacionales, 3\% Fisioterapeutas, 2\% Nutricionistas, $1 \%$ Sociólogo y Pedagogo y, $4 \%$ otras profesiones. Las orientaciones terapéuticas de los CAD, en las cuales se desempeñaban los profesionales de la muestra fue $34 \%$ de Comunidad terapéutica, $26 \%$ clínico psicológicos, $21 \%$ doce pasos, $18 \%$ psiquiátrico, $1 \%$ de teoterapia.
El profesional psicólogo estuvo representado en esta investigación por 35 personas, 21 (60\%) hombres y $14(40 \%)$ mujeres, quienes manifestaron desarrollar actividades asistenciales (49\%), actividades asistenciales y administrativas (23\%), educativas (17\%), actividades investigativas (6\%) y administrativas (5\%). El $97 \%$ promueve el desarrollo emocional, $85 \%$ desarrolla actividades de orientación e información, $82 \%$ establece el tratamiento a desarrollar, $80 \%$ establece estrategias comunicacionales con la familia, $77 \%$ realiza talleres y actividades de grupo.

El profesional médico en este estudio estuvo representado por 32 personas, 17 (53\%) hombres y 15 (47\%) mujeres, quienes manifesaron desarrollar actividades asistenciales en $34 \%$ de los casos, actividades administrativas y asistenciales en $31 \%$, actividades educativas $19 \%$ y actividades administrativas $16 \%$. El $87 \%$ de los médicos establece el tratamiento a desarrollar, 84\% realiza consulta individual, $81 \%$ desarrolla actividades de evaluación y seguimiento del tratamiento y de la institución y, 67\% realiza remisión de los pacientes

El profesional de enfermería estuvo representado en este estudio por 7 personas, $85 \%$ mujeres y $15 \%$ hombres, quienes manifestaron, en el $43 \%$ de los casos el desarrollo de actividades administrativas, $29 \%$ actividades asistenciales y administrativas, 14\% actividades educativas y $14 \%$ asistenciales. El $100 \%$ de los enfermeros manifiesta promover el autocuidado, $86 \%$ desarrolla actividades de evaluación o seguimiento,71\% realiza talleres y actividades en grupo y actividades de promoción de la salud. El $43 \%$ de las interacciones se realiza de forma grupal o individual y $14 \%$ de las dos formas.

El profesional de terapia ocupacional estuvo representado en esta investigación por cinco personas, 3 (60\%) hombres y 2 (40\%) mujeres, quienes manifestaron realizar (40\%) actividades asistenciales, $40 \%$ actividades administrativas y asistenciales y el $20 \%$ actividades administrativas. El 100\% desarrolla talleres y actividades grupales, $80 \%$ realiza consulta individual, $80 \%$ desarrolla actividades lúdicas y de prevención y, $80 \%$ promueve relaciones interpersonales en la comunidad.

El profesional de trabajo social se encontró representado en esta investigación por tres mujeres, quienes realizan actividades asistenciales (100\%), con este mismo porcentaje se documentó la promoción del desarrollo social, así como talleres y actividades grupales, en igual porcentaje se desarrollaban consulta 
individual, el 67\% desarrollaba actividades de promoción de la salud y, 67\% promovía el auto cuidado.

El profesional de fisioterapia estuvo representado en esta investigación por 3 personas, 2 (67\%) hombres y 1 (33\%) mujer. El 67\% manifestó desarrollar actividades asistenciales y administrativas y el 33\% actividades de tipo administrativo educativas. El $100 \%$ refirió promover el desarrollo físico, así como actividades de evaluación y seguimiento, también refirieron promover el auto cuidado en este mismo porcentaje $y, 67 \%$ desarrollaba actividades lúdicas y realizaban actividades de promoción.

El profesional de nutrición estuvo representado por $2(100 \%)$ mujeres quienes refirieron realizar actividades asistenciales y promover el desarrollo físico y el autocuidado; también desarrollaban consultas individuales de evaluación y seguimiento.

El profesional de sociología estuvo representado por una profesional, quien manifestó desarrollar actividades de administración y educación. Su papel está orientado a promover el desarrollo social y de formación a la familia y el paciente, mediante el desarrollo de estrategias comunicacionales, de rehabilitación y reinserción.

El profesional en pedagogía estuvo representado por un profesional masculino, quien manifestó desarrollar actividades de tipo administrativo y asistencial, su actuación estaba orientada a la rehabilitación y reinserción y al desarrollo social, mediante actividades lúdicas, talleres e interacciones grupales.

\section{Discusión}

La creciente situación, de uso y abuso de las drogas en varios países ${ }^{(10-13)}$ y en particular en Colombia(1-5), consecuente con un cambio en la dinámica de la producción, tráfico y consumo, ha determinado que se requiera mayor atención en la orientación política y normativa al respecto. Los entes estatales están ubicando ahora sus esfuerzos hacia el control de la demanda y no solo al desarrollo de políticas y acciones contra la oferta. Con el fin de responder a esta realidad, se evidencia la necesidad de incrementar el número y la calidad de atención de los Centros de Atención de Drogas (CAD), los cuales están dirigidos normativamente desde el punto de vista administrativo, asistencial y técnico por resoluciones y políticas emanadas del Ministerio de Protección Social.

El grupo de profesionales que participaron como muestra, tuvieron una edad promedio de 31 años, y un tiempo de experiencia laboral de 1 a 3 años, lo que evidencia un grupo joven, en plena etapa de productividad laboral y profesional, con predominio del género masculino y de la profesión de psicología, seguido por el profesional médico y la enfermera. Se resalta el hecho de haberse encontrado profesionales de otras disciplinas como terapia ocupacional, terapia física, nutricionista, trabajo social y pedagogos dentro del equipo de atención, estos resultados coinciden con los hallazgos del Ministerio de Protección Social del año 2004(5)

Se encontró que el tiempo completo, es decir 40 horas a la semana, así como la contratación por prestación de servicios, son los que predominan en las relaciones laborales de este grupo. Esto hallazgos son coincidentes con los Ministerio de Protección Social(5) y demuestran el cambio hacia tipos de contratación con menos garantías y estabilidad laboral - con el consiguiente deterioro de la calidad de vida de estos profesionales - los niveles de remuneración recibidos por el trabajo desarrollado mostraron un incremento respecto a lo encontrado en el año 2004(5); esto puede interpretarse de dos maneras: primero, como positivo debido a que se evidencia una mejoría neta en el salario que reciben los profesionales; por otro lado podría ser interpretado como cambios en la contratación, es decir contratos a término fijo, que implican el pago de prestaciones laborales incluidas en el salario y no como un incremento real del mismo.

Se evidenció que los profesionales, en más de la mitad de los casos, no tenían formación posgraduada en el área del fenómeno de las drogas; esto evidencia la necesidad de trabajar en la actualización del recurso humano que atiende a las personas con adicción en nuestro país, y que al ser enunciado como una estrategia para construir capacidad de respuesta, dentro de la Política de Reducción del Consumo(6), hace necesario la puesta en marcha dentro del eje operativo de Superación, determinado por el Ministerio de la Protección Social. Los profesionales manifiestan tener interacciones con los usuarios de tipo individual en el 93\% de los casos, esto continua demostrando que la visión de la terapia todavía no contempla a la familia como elemento fundamental del proceso.

La orientación terapéutica que predominó fue la de comunidad terapéutica, lo que es referido también en el documento de tratamiento de La Organización de Naciones Unidas(1). Los usuarios comparecen, principalmente, a la institución por presentar problemas de adicción a sustancias psicoactivas como el alcohol, el cigarrillo y la marihuana, estas son las sustancias que se consumen más en el país, según lo referido por 
el estudio Nacional de Salud Mental del año 2003(4). El número de pacientes atendidos por un profesional por semana fue de 11 a 15 en $30 \%$ de los casos. Los profesionales manifiestan que cuentan con los recursos humanos y materiales suficientes para cumplir con sus labores, esto debe ser visto como una fortaleza que debe mantenerse y apoyarse. Es importante señalar que los profesionales manifestaron que existe déficit del personal de enfermería en las instituciones, aspecto que ya había sido señalado en el estudio del año 2004 del Ministerio de protección social(5).

Con relación a las actividades y el tipo de interacciones que permitieron establecer el papel desempeñado por los profesionales, se evidenció que los papeles encontrados en la mayoría de los casos, están en concordancia con lo referido en la literatura.

El psicólogo fue el profesional más frecuente en los CAD, su papel se orienta a la promoción de la salud y al tratamiento de situaciones que alteran la salud emocional y mental a través de actividades asistenciales, mediante interacciones grupales que promueven la comunicación, la información y la orientación. El profesional en psicología está orientado a desarrollar apoyo emocional tanto al paciente como a su familia. En tanto que en la ley 58 de 1983 por la cual se reglamenta la profesión de psicólogo, en Colombia(14), se establece que son funciones del Psicólogo titulado, entre otras, la utilización de métodos y técnicas psicológicas con los siguientes objetivos; investigación básica y aplicada, docencia, diagnóstico psicológico, tratamiento psicológico, orientación y selección vocacional y profesional, análisis y modificación del comportamiento individual o grupal y, profilaxis psicológica.

El profesional médico ocupó el segundo lugar en frecuencia, su papel desempeñado, primordialmente, se evidencia por actividades de tipo asistencial que se orientan a establecer el diagnóstico y el tratamiento de las condiciones de la enfermedad o alteración del estado de salud física y mental, a través de la realización de consulta individual o la remisión apropiada en los casos necesarios. El médico "es el profesional "puerta de entrada" por que reciben al paciente en la institución, realiza el diagnóstico, establece el plan terapéutico y realiza las interconsultas necesarias ${ }^{(15)}$.

El papel del profesional en terapia ocupacional está orientado al desarrollo de actividades asistenciales y administrativas, mediante interacciones de tipo grupal con metodologías lúdicas y de talleres para promover las relaciones interpersonales y la prevención de la enfermedad. Es necesario señalar que en las funciones relacionadas con la rehabilitación y la inserción social no fueron evidentes en este grupo y al identificar - que la terapia ocupacional es la ciencia que tiene como fin facilitar la recuperación y reinserción de aquellas personas que sufren algún tipo de discapacidad física, psíquica o social, como lo señala el perfil del profesional de la Universidad nacional de Colombia - este hallazgo señala que este profesional no tiene su papel coherente con el área de estudio y formación.

El papel del trabajador social está relacionado con la promoción de aspectos de desarrollo social, así como el auto cuidado y la promoción de la salud mediante talleres y actividades grupales y de consulta individual. El papel es el de educador social(16).

El papel del fisioterapeuta está orientado a promover el desarrollo físico y el auto cuidado mediante actividades lúdicas de interacción grupal e individual. En el estudio de Saar, se encontró que este profesional se encarga de la rehabilitación motora, respiratoria y social, lo que concuerda con lo encontrado.

El papel del nutricionista está relacionado con la promoción del desarrollo físico y el autocuidado a través de consultas individuales de evaluación y seguimiento. En otra investigación se encontró que este profesional es considerado como auxiliar del médico y tiene por función determinar las necesidades dietéticas de las

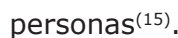

El papel del profesional de sociología está orientado a promover el desarrollo social y de formación de la familia y del paciente, mediante el desarrollo de estrategias comunicacionales, de rehabilitación y reinserción. El sociólogo se encarga del estudio de la vida social humana, de los grupos y sociedades(17).

El papel del pedagogo está orientado a la rehabilitación y reinserción y al desarrollo social, mediante actividades lúdicas, talleres e interacciones grupales. El objeto de la pedagogía es sistematizar la actividad humana conductora de las acciones educativas y de formación, en este caso a fin de lograr habilidades diversas para enfrentar los retos que impone el adaptarse a nuevos desempeños sociales, laborales y familiares $^{(18)}$.

El profesional de enfermería se encontró en el tercer lugar en frecuencia en los CAD. Su papel está relacionado, principalmente, con el desarrollo de actividades administrativas; su interacción con los usuarios está dirigida a la promoción del autocuidado y la salud mediante actividades de evaluación y talleres con aproximaciones de tipo grupal ocasionalmente e individual principalmente. El desempeño de la 
enfermería en el área de salud mental, está orientada a la administración y a la administración intermediaria para los otros profesionales, además, estos son los que menos atenciones directas realizan ${ }^{(19)}$, lo cual es evidente en el resultado obtenido en el presente estudio; esta es una situación lamentable porque es la enfermera la llamada a tener un contacto más cercano con las personas a las cuales cuida en virtud del carácter interactivo e integral del cuidado.

Al comparar las actividades desarrolladas por los profesionales y las enfermeras y teniendo como base lo referido en la normatividad creada para regular los Centros de Atención en Drogas, se encontró que, respecto a la actividad de dar orientación e información a la familia y el usuario, más de la mitad lo hacen. Su orientación como enfermeras les permite desarrollar esta importante función en el ámbito de las instituciones de atención en ambulatorios, aspecto que concuerda con lo referido en el manual sobre el tratamiento de abuso de drogas, el cual refiere que la enfermera presta asistencia a familia que se encuentra afectada por el problema de abuso de sustancias y puede proporcionar información y asesoramiento de apoyo(20-21).

\section{Conclusiones}

El grupo interdisciplinario se encontró conformado por psicólogos, médicos, enfermeros, trabajadores sociales, fisioterapeutas, terapeutas ocupacionales, sociólogos y pedagogos, jóvenes y de género masculino primordialmente, los cuales manifestaron desarrollar actividades administrativas, educativas y asistenciales dentro de los CAD de la ciudad. Las actividades asistenciales se desarrollaban mediante interacciones de tipo individual en la mayoría de los casos. Los profesionales que, en mayor número, se encuentran en los CAD son los psicólogos, que se desempeñan principalmente en comunidades terapéuticas y de orientación psicoterapéutica. Los papeles desempeñados por los profesionales coinciden con la visión psicosocial esperada, respecto a las conductas y contenidos de las diferentes profesiones con excepción de la salud ocupacional.

Se constató que los enfermeros y enfermeras que trabajaban en los CAD, enfatizaron sus funciones en el ámbito administrativo, predominantemente en los aspectos de promoción y prevención, y muy poco en la rehabilitación y reinserción social.

Se evidencia que la normatividad vigente de la "Condiciones de habilitación para centros de atención en drogadicción y servicios de farmacodependencia", puede retomar lo enunciado en la resolución 196 de 2002, respecto a actividades a desarrollar en los CAD, puesto que ésta ultima determina criterios claros y concretos de lo esperado en la atención de las personas que asisten a los centros CAD. Es necesario operacionalizar lo contenido en la política de reducción del consumo de sustancias psicoactivas bajo la perspectiva de lo que cada profesión puede ofrecer desde el núcleo de su disciplina.

\section{Agradecimientos}

Agradecemos a la Comisión Interamericana para el Control del Abuso de Drogas/CICAD de la Secretaria de Seguridad Multidimensional/SSM de la Organización de los Estados Americanos/OEA, la Secretaria Nacional de Políticas sobre Drogas/SENAD do Gabinete de Seguridad Institucional/Brasil, la Escuela de Enfermería de Ribeirao Preto de la Universidad de Sao Paulo y Centro Colaborador de la Organización Mundial de la Salud para el Desarrollo de la Investigación en Enfermería, la población representada en los estudios de investigación, bien como a las autoridades de las universidades representadas por los participantes del Programa En-Line de Especialización en Investigación sobre el Fenómeno de las Drogas - PREINVEST, periodos 2005, 2006, 2007 y 2008.

\section{Referencias}

1.Naciones Unidas. Oficina contra la droga y el delito. Jóvenes y drogas en países sudamericanos: un desafío para las políticas públicas. Primer estudio comparativo sobre uso de drogas en población escolar secundaria. [Libro en internet] Lima Perú; 2006. [acceso 14 de agosto de 2008]. Disponible en: www.cicad.oas. org/oid/new/statistics/siduc/infofinal_estudio_comparativo.pdf

2. República de Colombia. Ministerio del interior y justicia. Dirección Nacional de estupefacientes. Observatorio de drogas de Colombia. Estadísticas Consumo.2008. [Sede web]. [acceso 14 de agosto de 2008]. Disponible en: http://www.dne.gov.co/ tools/marco.php?idcategoria $=633$
3. Osorio EA. Ortega de Medina NM, Pillon, SC. Factores de riesgo asociados al uso de drogas en estudiantes adolescentes. Rev. Latino-Am. Enfermagem 2004; mar./abr.12(spe):369375. Disponible en: http://www.scielo.br/pdf/rlae/v12nspe/ v12nspea11.pdf

4. Dirección Nacional de Estupefacientes. Observatorio de Drogas de Colombia: Acciones y Resultados 2003. Bogotá DC. Subdirección Estratégica y de Investigaciones/DNE. 2003. [libro en internet]. [Acceso el 14 de agosto de 2008] Disponible en http://www.dne.gov.co/?idcategoria $=834$ 
5. Ministerio de Protección social. [Sede web] Encuesta Nacional sobre sustancias psicoactivas. 2004. [Acceso el 14 de agosto de 2008]. Disponible en: http://www.minproteccionsocial.gov.co/ VBeContent/NewsDetail.asp?ID=14743\&IDCompany $=3$

6. República de Colombia. Ministerio del interior y justicia. Dirección Nacional de estupefacientes. Observatorio de drogas de Colombia. Política Nacional para la reducción del consumo sustancias psicoactivas y su impacto. Mejía IE, Parra A Bogotá. 2007. [acceso el 14 de agosto de 2008].Disponible en: http:// odc.dne.gov.co/IMG/pdf/Resumen_20Ejecutivo_20PNRCSPA.pdf 7. Aritzeta A, Ayestarán S. Aplicabilidad de la teoría de los roles de equipo de Belbin: un estudio comparativo con equipos de trabajo. Rev Psicol Gral y Aplic 2003 jan./fev./mar.;56(1):6175.Disponible en: http://dialnet.unirioja.es/servlet/ articulo? codigo $=649325$.

8. Wright MG, Chisman A. A saúde internacional, o fenômeno das drogas e a profissão de enfermagem na América Latina. Texto \& Contexto Enferm. 2002 abr./jun.; 13(2):264.

9. Crabtree, BF, Miller, WLDoing Qualitative Research. Research Methods for Primary Care. v. 13. Newbury Park, California: Sage Publishing; 1992.

10. Mendes IAC, Marziale MHP. Desarrollando competencias para la reducción de la demanda de drogas Rev. Latino-Am. Enfermagem 2008 jul./ago;16(spe):505-6. Disponible en: http://www.scielo.br/pdf/rlae/v16nspe/01.pdf.

11. Castillo BAA, Marziale MHP, Castillo MMA, Facundo FRG, Meza MVG. Situaciones de la vida estresantes, uso y abuso de alcohol y drogas en adultos mayores de Monterrey, México. Rev. LatinoAm. Enfermagem. 2008 jul./ago;16(spe):509-15. Disponible en: http://www.scielo.br/pdf/rlae/v16nspe/02.pdf.

12. Castillo CO, Costa MCS. Significados del consumo de alcohol en familias de una comunidad pobre venezolana. Rev. LatinoAm. Enfermagem. 2008 jul./ago;16(spe):535-42. Disponible en: http://www.scielo.br/pdf/rlae/v16nspe/06.pdf.
13. Gil HLB, Mello DF, Ferriani MGC, Silva MAI. Opiniones de los adolescentes escolares sobre consumo de drogas: un estudio de caso en Lima, Perú. Rev. Latino-Am. Enfermagem. 2008 jul./ ago; 16(spe):551-7. Disponible en: http://www.scielo.br/pdf/ rlae/v16nspe/08.pdf.

14. Reglamentación de la profesión de psicólogo Ley 58. Congreso de la república de Colombia. (Diciembre 28 de 1983). 15. Saar Sandra Regina da Costa, Trevizan Maria Auxiliadora. Los roles profesionales de un equipo de salud: la visión de sus integrantes. Rev. Latino-Am. Enfermagem. 2007 jan./ fev.;15(1):106-12. Disponible en: http://www.scielo.br/pdf/ rlae/v15n1/pt_v15n1a16.pdf

16. Mazibuko F, Gray M. Social Work Professional Associations in South Africa. International Social Work 2004; jan; 47(1):129-42. Available from: http://isw.sagepub.com/cgi/reprint/47/1/129 17. Giddens A. La Constitución de la Sociedad. Argentina: Ed Amorrortu; 1995.

18 Gómez Mendoza M. Introducción a la Didáctica de la Filosofía. Colombia: Editorial Papiro; 2003.

19. Oliveira $A B$, Alessi NP. El trabajo de enfermería en salud mental: contradicciones y potencialidades actuales. Rev. LatinoAm. Enfermagem. 2003 maio/jun.; 11(3):330-40. Disponible en: http://www.scielo.br/pdf/rlae/v11n3/16543.pdf

20. Naciones Unidas, Oficina contra la droga y el delito. Abuso de Drogas: tratamiento y rehabilitación. Guía practica de planificación y planeación. Nueva York 2003. [Libro en internet] [acceso el 14 de agosto de 2008]. Disponible en: www.unodc. org/docs/treatment/Guide_S.pdf

21. Motta, IE. Ministerio de protección social. Diagnostico situacional: Instituciones de tratamiento, rehabilitación y reincorporación social a consumidores de Psicoactivos en Colombia. Bogotá. 2004. 